\title{
Subspace Clustering
}

\author{
with the Multivariate- $t$ Distribution
}




\title{
SUBSPACE CLUSTERING WITH THE MULTIVARIATE-T DISTRIBUTION
}

BY

ANGELINA PESEVSKI, B.Sc.

\author{
A THESIS \\ SUBMITTED TO THE DEPARTMENT OF MATHEMATICS \& STATISTICS \\ AND THE SCHOOL OF GRADUATE STUDIES \\ OF MCMASTER UNIVERSITY
}

IN PARTIAL FULFILMENT OF THE REQUIREMENTS

FOR THE DEGREE OF MASTER OF SCIENCE

(c) Copyright by Angelina Pesevski, July 2017

All Rights Reserved 
Master of Science (2017)

(Mathematics \& Statistics)
McMaster University

Hamilton, Ontario, Canada

TITLE: $\quad$ Subspace Clustering

with the Multivariate- $t$ Distribution

AUTHOR:

Angelina Pesevski

B.Sc., (Mathematics and Statistics)

McMaster University, Hamilton, Canada

SUPERVISOR:

Dr. Paul D. McNicholas

Dr. Brian C. Franczak

NUMBER OF PAGES: ix, 39 
To my loving and supporting partner, Kiril, as well as the individuals who gave me this opportunity, my parents, Liljana and Bobi. 


\section{Abstract}

Clustering procedures suitable for the analysis of very high-dimensional data are needed for many modern data sets. One model-based clustering approach called high-dimensional data clustering (HDDC) uses a family of Gaussian mixture models to model the sub-populations of the observed data, i.e., to perform cluster analysis. The HDDC approach is based on the idea that high-dimensional data usually exists in lower-dimensional subspaces; as such, the dimension of each subspace, called the intrinsic dimension, can be estimated for each sub-population of the observed data. As a result, each of these Gaussian mixture models can be fitted using only a fraction of the total number of model parameters. This family of models has gained attention due to its superior classification performance compared to other families of mixture models; however, it still suffers from the usual limitations of Gaussian mixture modelbased approaches. Herein, a robust analogue of the HDDC approach is proposed. This approach, which extends the HDDC procedure to include the mulitvariate- $t$

distribution, encompasses 28 models that rectify one of the major shortcomings of the HDDC procedure. Our $t$ HDDC procedure is fitted to both simulated and real data sets and is compared to the HDDC procedure using an image reconstruction problem that arose from satellite imagery of Mars' surface. 


\section{Acknowledgements}

First and foremost, I'd like to thank my supervisor Professor Paul D. McNicholas. Thank you for giving me this opportunity and teaching me to push myself to new limits. I will forever be grateful for your encouraging words that got me to where I am today.

I would also like to thank Dr. Brian C. Franczak for all the guidance and support throughout this project. I cannot even begin to express my gratitude for all of the hours you have dedicated to me, for the daily coding sessions and the occasional pep talk. You have been a great mentor and friend, and I am glad that I will have the pleasure to continue working under your supervision.

To my parents, Lile and Bobi, you have dedicated your lives to your children's academic and personal success, and for that I will be forever thankful. Even if I become half the parent and friend you both are, I know that I will have succeeded in my life. Thank you for giving me a chance at this life and for always believing in me.

To my sister, thank you for all the encouragement and positivity you radiate on a daily basis. I have had an easier life because you always got to go first and I learned from your mistakes, especially when it comes to education.

Last but definitely not least, I would like to thank my partner, Kire. You have always given me that burst of energy when I was frustrated with my code or when I 
was in a writing rut. You have given me confidence and made me understand that I can accomplish anything I set my mind to. The following years of studies are not going to be easy, but I am welcoming them with open arms, all because of you. 


\section{Contents}

Abstract $\quad$ iv

Acknowledgements $\quad$ v

Bibliography ................................ vii

1 Introduction 1

2 Background 3

2.1 Normal-Variance Mixtures . . . . . . . . . . . . . . . . . . . 3

2.2 Finite Mixture-Models . . . . . . . . . . . . . . . . . . . 4

2.3 Gaussian Mixture-Model . . . . . . . . . . . . . . . . . 4

2.4 Mixtures of Multivariate-t Distributions . . . . . . . . . . . . 5

2.5 Gaussian Parsimonious Clustering Models . . . . . . . . . . . 6

2.6 tEIGEN Models . . . . . . . . . . . . . . . . . . . . 7

2.7 Parsimonious Gaussian Mixture Models . . . . . . . . . . . . . . . 8

2.8 Mixture of Multivariate-t Factor Analyzers . . . . . . . . . . . . . . . 9

2.9 High-Dimensional Data Clustering . . . . . . . . . . . . . . . 10

2.10 Model Selection and Performance Assesment . . . . . . . . . . . . . . 11 
3 Methodology 13

3.1 Formulating the $t$ HDDC Approach . . . . . . . . . . . . . . 14

3.2 Parameter Estimation . . . . . . . . . . . . . . . 15

3.2.1 The E-step . . . . . . . . . . . . . . . 16

3.2 .2 The CM-steps . . . . . . . . . . . . . . . . . . . . 17

3.3 Convergence . . . . . . . . . . . . . . . . . . . . 19

4 Application $\quad 21$

4.1 Computational Considerations . . . . . . . . . . . . . . . . 21

4.2 Simulation Studies . . . . . . . . . . . . . . . . . . . 22

4.3 Fisher's Irises . . . . . . . . . . . . . . . . . . . . . 23

4.4 Italian Wines . . . . . . . . . . . . . . . . . 25

4.5 Martian Surface . . . . . . . . . . . . . . 26

5 Conclusions and Future Work $\quad 29$

$\begin{array}{ll}\text { A Cost Function Derivations } & 31\end{array}$

$\begin{array}{ll}\text { Bibliography } & 33\end{array}$ 


\section{List of Figures}

4.1 Pairs and density plots of the first three dimensions of one multivariate$t$ simulated data set, coloured by true groups. . . . . . . . . .

4.2 The iris data coloured by the classes found by the best fitting $t$ HDDC model and projected onto the one-dimensional spaces for each compo-

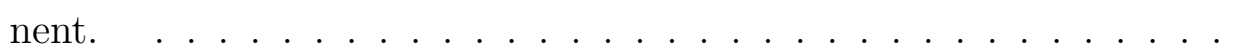

4.3 Pairs and density plots of the Italian wines data colored by classes found by the best fitting $t$ HDDC model and projected onto the fourdimensional subspace. . . . . . . . . . . . . . . .

4.4 Three images of the Martian surface constructed using spectral data collected by the OMEGA instrument (top), the parameter estimates of the best-fitting $t$ HDDC model (middle) and the parameter estimates of the best-fitting HDDC model (bottom). . . . . . . . . . . 


\section{Chapter 1}

\section{Introduction}

Cluster analysis refers to the practice of using statistical approaches to detect subgroups within a given data set. These subgroups can represent a physical attribute not described by the given explanatory variables, e.g., gender, income tax bracket or blood type, which can reveal important relationships among the observed data and may be a crucial component in the effective analysis of a given data set. Due to their construction, finite mixture models are very useful when modelling data that contain a finite collection of sub-populations because each component of the model can be used to represent one of these sub-populations. Reviews of the application of finite mixture models for clustering are given by Fraley and Raftery (2002); Bouveyron and Brunet-Saumard (2014) and McNicholas (2016b), and extensive details can be found in the monographs by McLachlan and Peel (2000) and McNicholas (2016a).

The current influx of information from, e.g., social media sources, has resulted in many modern data sets having the characteristics of big data (Puts et al., 2015). As such, there is an increasing need for statistical methods that can handle these large data sets. In Chapter 2, we provide a review of popular finite mixture-models for 
handling big data sets, specifically those with large dimension, and highlight a highdimensional data clustering (HDDC) approach recently introduced by Bouveyron et al. (2007). This approach has gained attention due to its parsimonious parameter estimation scheme. It has been shown to work well for very high-dimensional real data of more than 200 variables. However, it still suffers from the usual limitations of the Gaussian mixture model based approaches, e.g., it is not robust to outliers. The focus of this thesis is the expansion of this HDDC approach to include a multivariate distribution that is robust to outliers.

A formal outline of this thesis follows: in Chapter 2, we provide background information on finite mixture-models and summarize related work, in Chapter 3, we outline the derivation of a multivariate- $t$ high-dimensional data clustering $(t H D D C)$ approach and describe a parameter estimation scheme to fit the resulting models, in Chapter 4 we assess the classification performance of this novel family of models using a simulation study and three real data sets, and in Chapter 5 we conclude with a discussion and suggestions for future work. 


\section{Chapter 2}

\section{Background}

\section{$2.1 \quad$ Normal-Variance Mixtures}

Define a real random variable $U$ following a probability distribution $H$, where $H \in$ $[0, \infty)$ and a $p$-dimensional random variable $\mathbf{X}$. We can say that $\mathbf{X}$ follows a normalvariance mixture if $\mathbf{X} \mid U=u$ follows a multivariate Gaussian distribution with mean $\boldsymbol{\mu}$ and covariance matrix $u \boldsymbol{\Sigma}$, where $\boldsymbol{\mu}$ is a location parameter and $\boldsymbol{\Sigma}$ is a $p \times p$ covariance matrix (Barndorff-Nielsen et al., 1982). Formally, a random vector $\mathbf{X}$ arising from a normal-variance mixture has density

$$
f(\mathbf{x} \mid \boldsymbol{\mu}, \boldsymbol{\Sigma})=\int_{0}^{\infty} \phi_{p}(\mathbf{x} \mid u ; \boldsymbol{\mu}, u \boldsymbol{\Sigma}) h(u) d u
$$

where $\phi_{p}(\mathbf{X} \mid u ; \boldsymbol{\mu}, u \boldsymbol{\Sigma})$ is the density function of a $p$-variate Gaussian distribution with location parameter $\boldsymbol{\mu}$ and covariance matrix $u \boldsymbol{\Sigma}$. There are several distributions that arise as mixtures of Normal distributions, one being the multivariate- $t$ distribution (Kotz and Nadarajah, 2004). 


\section{$2.2 \quad$ Finite Mixture-Models}

The density of a parametric finite mixture distribution is

$$
f(\mathbf{x} \mid \boldsymbol{\vartheta})=\sum_{g=1}^{G} \pi_{g} p_{g}\left(\mathbf{x} \mid \boldsymbol{\theta}_{g}\right),
$$

where $\pi_{g}>0$, such that $\sum_{g=1}^{G} \pi_{g}=1$, are called mixing proportions, $p_{g}\left(\mathbf{x} \mid \boldsymbol{\theta}_{g}\right)$ are the component densities and $\boldsymbol{\vartheta}=\left(\pi_{1}, \ldots, \pi_{G}, \boldsymbol{\theta}_{1}, \ldots, \boldsymbol{\theta}_{G}\right)$ is a vector containing the model parameters. Herein, we follow convention and refer to the application of finite mixture models for clustering as model-based clustering.

As described in McNicholas (2016a), there are many different definitions of a cluster. A common definition discussed by Wolfe (1963) is one where a cluster is described as a group of observations that are more similar to each other than to observations outside that particular group. A more rigorous definition, given by Wolfe (1963), requires the use of finite mixture models and defines a cluster as being one of the components of a mixture of distributions.

\subsection{Gaussian Mixture-Model}

Each component of the density of the general finite mixture model, given in (2.2), can be specified to be any univariate or multivariate probability distribution. Until the last decade or so, the majority of work on model-based clustering using multivariate component densities focused on the Gaussian mixture-model. A random variable $\mathbf{X} \in \mathbb{R}^{p}$ arising from a multivariate Gaussian distribution with mean $\boldsymbol{\mu}$ and covariance 
matrix $\boldsymbol{\Sigma}$ has density

$$
\phi_{p}(\mathbf{x} \mid \boldsymbol{\mu}, \boldsymbol{\Sigma})=\frac{1}{(2 \pi)^{p / 2}|\boldsymbol{\Sigma}|^{1 / 2}} \exp \left\{-\frac{1}{2}(\mathbf{x}-\boldsymbol{\mu})^{\prime} \boldsymbol{\Sigma}^{-1}(\mathbf{x}-\boldsymbol{\mu})\right\} .
$$

It follows that the density of a mixture of multivariate Gaussian distributions is

$$
f\left(\mathbf{x} \mid \pi_{g}, \boldsymbol{\mu}_{g}, \boldsymbol{\Sigma}_{g}\right)=\sum_{g=1}^{G} \pi_{g} \frac{1}{(2 \pi)^{p / 2}\left|\boldsymbol{\Sigma}_{g}\right|^{1 / 2}} \exp \left\{-\frac{1}{2}\left(\mathbf{x}-\boldsymbol{\mu}_{g}\right)^{\prime} \boldsymbol{\Sigma}_{g}^{-1}\left(\mathbf{x}-\boldsymbol{\mu}_{g}\right)\right\},
$$

where $\pi_{g}$ is defined for (2.2), and $\boldsymbol{\mu}_{g}$ and $\boldsymbol{\Sigma}_{g}$ are the component location parameter and component covariance matrix, respectively. In total, the general Gaussian mixture model has $(G-1)+G p+G p(p+1) / 2$ free parameters.

\subsection{Mixtures of Multivariate- $t$ Distributions}

One of the first notable departures from Gaussianity was provided by Peel and McLachlan (2000), who utilized mixtures of multivariate- $t$ distributions for clustering. As the name suggests, mixtures of multivariate-t distributions assume that each sub-population of the observed data follow the multivariate- $t$ distribution. Formally, the density of a mixture of multivariate- $t$ distribution is formulated by writing the component density in (2.2) as

$$
p_{g}\left(\mathbf{x} \mid \boldsymbol{\theta}_{g}\right)=f_{t}\left(\mathbf{x} \mid \boldsymbol{\mu}_{g}, \boldsymbol{\Sigma}_{g}, \nu_{g}\right)=\frac{\Gamma\left[\left(\nu_{g}+p\right) / 2\right]\left|\boldsymbol{\Sigma}_{g}\right|^{-1 / 2}\left(\pi \nu_{g}\right)^{-p / 2}}{\Gamma\left[\nu_{g} / 2\right]\left[1+\delta\left(\mathbf{x}, \boldsymbol{\mu}_{g} \mid \boldsymbol{\Sigma}_{g}\right) / \nu_{g}\right]^{\left(\nu_{g}+p\right) / 2}},
$$

where $\Gamma(\cdot)$ is the Gamma function, $p$ is the number of dimensions in the observed data set, $\boldsymbol{\mu}_{g}$ is the component location parameter, $\boldsymbol{\Sigma}_{g}$ is the component covariance 
matrix, $\nu_{g}$ parameterizes the degrees of freedom in each component, and

$$
\delta\left(\mathbf{x}, \boldsymbol{\mu}_{g} \mid \boldsymbol{\Sigma}_{g}\right)=\left(\mathbf{x}-\boldsymbol{\mu}_{g}\right)^{\prime} \boldsymbol{\Sigma}_{g}^{-1}\left(\mathbf{x}-\boldsymbol{\mu}_{g}\right)
$$

is the squared Mahalanobis distance between $\mathbf{x}$ and $\boldsymbol{\mu}_{g}$ for $g=1, \ldots, G$. Despite rectifying a well known shortcoming of the Gaussian mixture model by formulating a model that is robust to outliers, mixtures of multivariate- $t$ distributions have only gained popularity in the last few years (see McLachlan et al., 2007; Andrews and McNicholas, 2011a,b; Baek and McLachlan, 2011; Steane et al., 2012; Andrews and McNicholas, 2012; Lin et al., 2014, for examples).

\subsection{Gaussian Parsimonious Clustering Models}

A family of mixture models emerges when we introduce constraints on the component densities. Some families of Gaussian mixture models are well established and widely used, e.g., the Gaussian Parsimonious Clustering Models (GPCMs; Celeux and Govaert, 1995) which arise from constraints being imposed on the eigen-decomposed covariance structure in a Gaussian mixture model. This eigen-decomposition is

$$
\boldsymbol{\Sigma}_{g}=\lambda_{g} \mathbf{D}_{g} \mathbf{A}_{g} \mathbf{D}_{g}^{\prime}
$$

where $\boldsymbol{\Sigma}_{g}$ is the component covariance matrix, $\lambda_{g}$ is a constant, $\mathbf{D}_{g}$ is a matrix of eigenvectors, and $\mathbf{A}_{g}$ is a diagonal matrix with $\left|\mathbf{A}_{g}\right|=1$ and entries proportional to the eigenvalues of $\boldsymbol{\Sigma}_{g}$. Applying a combination of the constraints: $\lambda_{g}=\lambda, \mathbf{A}_{g}=$ $\mathbf{A}, \mathbf{D}_{g}=\mathbf{D}, \mathbf{D}_{g}=\mathbf{I}, \mathbf{A}_{g}=\mathbf{I}$, where $\mathbf{I}$ is the identity matrix of the appropriate 
dimension, across the components of the Gaussian mixture model results in a family of fourteen models and allows for various shapes and sizes of clusters (see Table 2.1). In more than half of these fourteen models there are $\mathcal{O}\left(p^{2}\right)$ free parameters to be estimated; hence, with higher dimensions, it can be very computationally inefficient to use these models. The GPCMs are supported by the R packages mclust (Fraley and Raftery, 2002) and mixture (Browne et al., 2015).

Table 2.1: Nomenclature, component volume, shape and orientation, covariance structure, and number of free covariance parameters for each member of the GPCM family.

\begin{tabular}{lllllr}
\hline Model & Volume & Shape & Orientation & $\boldsymbol{\Sigma}_{g}$ & Free cov. parameters \\
\hline EII & Equal & Spherical & - & $\lambda \mathbf{I}$ & 1 \\
VII & Variable & Spherical & - & $\lambda_{g} \mathbf{I}$ & $G$ \\
EEI & Equal & Equal & Axis-Aligned & $\lambda \mathbf{A}$ & $p$ \\
VEI & Variable & Equal & Axis-Aligned & $\lambda_{g} \mathbf{A}$ & $p+G-1$ \\
EVI & Equal & Variable & Axis-Aligned & $\lambda \mathbf{A}_{g}$ & $p G-G+1$ \\
VVI & Variable & Variable & Axis-Aligned & $\lambda_{g} \mathbf{A}_{g}$ & $p G$ \\
EEE & Equal & Equal & Equal & $\lambda \mathbf{D A D}^{\prime}$ & $p(p+1) / 2$ \\
EEV & Equal & Equal & Variable & $\lambda \mathbf{D}_{g} \mathbf{A D}_{g}^{\prime}$ & $G p(p+1) / 2-(G-1) p$ \\
VEV & Variable & Equal & Variable & $\lambda_{g} \mathbf{D}_{g} \mathbf{A D}_{g}^{\prime}$ & $G p(p+1) / 2-(G-1)(p-1)$ \\
VVV & Variable & Variable & Variable & $\lambda_{g} \mathbf{D}_{g} \mathbf{A}_{g} \mathbf{D}_{g}^{\prime}$ & $G p(p+1) / 2$ \\
EVE & Equal & Variable & Equal & $\lambda \mathbf{D A} \mathbf{A}_{g} \mathbf{D}^{\prime}$ & $p(p+1) / 2+(G-1)(p-1)$ \\
VVE & Variable & Variable & Equal & $\lambda_{g} \mathbf{D} \mathbf{A}_{g} \mathbf{D}^{\prime}$ & $p(p+1) / 2+(G-1) p$ \\
VEE & Variable & Equal & Equal & $\lambda_{g} \mathbf{D} \mathbf{A D}^{\prime}$ & $p(p+1) / 2+(G-1)$ \\
EVV & Equal & Variable & Variable & $\lambda \mathbf{D}_{g} \mathbf{A}_{g} \mathbf{D}_{g}^{\prime}$ & $G p(p+1) / 2-(G-1)$ \\
\hline
\end{tabular}

\section{$2.6 \quad t$ EIGEN Models}

The multivariate- $t$ analog of the GPCM family for the mixtures of multivariate- $t$ distributions is the $t$ EIGEN family (Andrews and McNicholas, 2012). These models use the same eigen-decomposition as the GPCM family and therefore the same constraints mentioned above can be applied, in addition to $\nu_{g}=\nu$. It is important to note that in this case, it is not the covariance matrix that is decomposed, but rather 
the scale matrix. Furthermore, as explained in (Vrbik and Mcnicholas, 2014), the geometric interpretation of the component shapes in non-Gaussian mixture-models is not the same as with the Gaussian cases.

By combining the aforementioned constraints, a total of 28 different models are derived. In R, all 28 models are supported by the teigen package (Andrews and McNicholas, 2014; Andrews et al., 2017).

\subsection{Parsimonious Gaussian Mixture Models}

Another popular family of mixture models are the parsimonious Gaussian mixture models (PGMMs; McNicholas and Murphy, 2008). These models are an extension of the mixture of factor analyzers (Ghahramani and Hinton, 1997) whose component covariance matrices are written as

$$
\Sigma_{g}=\Lambda_{g} \Lambda_{g}^{\prime}+\Psi_{g}
$$

where $\boldsymbol{\Lambda}_{g}$ is a $p \times q$ loading matrix with $q<p$, and $\boldsymbol{\Psi}_{g}$ is a diagonal $p \times p$ matrix with positive entries for $g=1, \ldots, G$. By imposing constraints on $\boldsymbol{\Lambda}_{g}$ and $\boldsymbol{\Psi}_{g}$ across the components, McNicholas and Murphy (2008) introduced eight parsimonious models in which the number of free parameters is $\mathcal{O}(p)$ so that the number of covariance parameters grows linearly with dimension. For this reason, these models are more appropriate than the GPCMs for high-dimensional data. These models can be implemented via the pgmm package for R (McNicholas et al., 2015). Note: McNicholas 
and Murphy (2010) extended the PGMMs to include four new models by setting

$$
\Psi_{g}=\omega_{g} \boldsymbol{\Delta}_{g},
$$

where $\omega_{g} \in \mathbb{R}^{+}$and $\boldsymbol{\Delta}_{g}=\operatorname{diag}\left\{\delta_{1}, \delta_{2}, \ldots, \delta_{p}\right\}$ is a noise matrix, such that $\left|\boldsymbol{\Delta}_{g}\right|=1$ (see Table 2.2).

Table 2.2: Nomenclature, component covariance matrix structure, and number of free covariance parameters for each parsimonious Gaussian mixture models.

\begin{tabular}{|c|c|c|c|c|c|}
\hline \multicolumn{4}{|c|}{ PGMM Nomenclature } & \multirow[b]{2}{*}{ Component Covariance Matrix } & \multirow[b]{2}{*}{ Number of Free Scale Parameters } \\
\hline$\underline{\Lambda_{g}=\boldsymbol{\Lambda}}$ & $\boldsymbol{\Delta}_{g}=\boldsymbol{\Delta}$ & $\omega_{g}=\omega$ & $\boldsymbol{\Delta}_{g}=\mathbf{I}_{p}$ & & \\
\hline $\mathrm{C}$ & $\mathrm{C}$ & $\mathrm{C}$ & $\mathrm{C}$ & $\boldsymbol{\Sigma}_{g}=\boldsymbol{\Lambda} \boldsymbol{\Lambda}^{\prime}+\omega \mathbf{I}_{p}$ & {$[p q-q(q-1) / 2]+1$} \\
\hline $\mathrm{C}$ & $\mathrm{C}$ & $\mathrm{U}$ & $\mathrm{C}$ & $\boldsymbol{\Sigma}_{g}=\boldsymbol{\Lambda} \boldsymbol{\Lambda}^{\prime}+\omega_{g} \mathbf{I}_{p}$ & {$[p q-q(q-1) / 2]+G$} \\
\hline $\mathrm{U}$ & $\mathrm{C}$ & $\mathrm{C}$ & $\mathrm{C}$ & $\boldsymbol{\Sigma}_{g}=\boldsymbol{\Lambda}_{g} \boldsymbol{\Lambda}_{g}^{\prime}+\omega \mathbf{I}_{p}$ & $G[p q-q(q-1) / 2]+1$ \\
\hline $\mathrm{U}$ & $\mathrm{C}$ & $\mathrm{U}$ & $\mathrm{C}$ & $\boldsymbol{\Sigma}_{g}=\boldsymbol{\Lambda}_{g} \boldsymbol{\Lambda}_{g}^{\prime}+\omega_{g} \mathbf{I}_{p}$ & $G[p q-q(q-1) / 2]+G$ \\
\hline $\mathrm{C}$ & $\mathrm{C}$ & $\mathrm{C}$ & $\mathrm{U}$ & $\boldsymbol{\Sigma}_{g}=\boldsymbol{\Lambda} \boldsymbol{\Lambda}^{\prime}+\omega \boldsymbol{\Delta}$ & {$[p q-q(q-1) / 2]+p$} \\
\hline $\mathrm{C}$ & $\mathrm{C}$ & $\mathrm{U}$ & $\mathrm{U}$ & $\boldsymbol{\Sigma}_{g}=\boldsymbol{\Lambda} \boldsymbol{\Lambda}^{\prime}+\omega_{g} \boldsymbol{\Delta}$ & {$[p q-q(q-1) / 2]+[G+(p-1)]$} \\
\hline $\mathrm{U}$ & $\mathrm{C}$ & $\mathrm{C}$ & $\mathrm{U}$ & $\boldsymbol{\Sigma}_{g}=\boldsymbol{\Lambda}_{g} \boldsymbol{\Lambda}_{g}^{\prime}+\omega \boldsymbol{\Delta}$ & $G[p q-q(q-1) / 2]+p$ \\
\hline $\mathrm{U}$ & $\mathrm{C}$ & $\mathrm{U}$ & $\mathrm{U}$ & $\boldsymbol{\Sigma}_{g}=\boldsymbol{\Lambda}_{g} \boldsymbol{\Lambda}_{g}^{\prime}+\omega \boldsymbol{\Delta}_{g}$ & $G[p q-q(q-1) / 2]+[G+(p-1)]$ \\
\hline $\mathrm{C}$ & $\mathrm{U}$ & $\mathrm{C}$ & $\mathrm{U}$ & $\boldsymbol{\Sigma}_{g}=\boldsymbol{\Lambda} \boldsymbol{\Lambda}^{\prime}+\omega \boldsymbol{\Delta}_{g}$ & {$[p q-q(q-1) / 2]+[1+G(p-1)]$} \\
\hline $\mathrm{C}$ & $\mathrm{U}$ & $\mathrm{U}$ & $\mathrm{U}$ & $\boldsymbol{\Sigma}_{g}=\boldsymbol{\Lambda} \boldsymbol{\Lambda}^{\prime}+\omega_{g} \boldsymbol{\Delta}_{g}$ & {$[p q-q(q-1) / 2]+G p$} \\
\hline $\mathrm{U}$ & $\mathrm{U}$ & $\mathrm{C}$ & $\mathrm{U}$ & $\boldsymbol{\Sigma}_{g}=\boldsymbol{\Lambda}_{g} \boldsymbol{\Lambda}_{g}^{\prime}+\omega \boldsymbol{\Delta}_{g}$ & $G[p q-q(q-1) / 2]+[1+G(p-1)]$ \\
\hline $\mathrm{U}$ & $\mathrm{U}$ & $\mathrm{U}$ & $\mathrm{U}$ & $\boldsymbol{\Sigma}_{g}=\boldsymbol{\Lambda}_{g} \boldsymbol{\Lambda}_{g}^{\prime}+\omega_{g} \boldsymbol{\Delta}_{g}$ & $G[p q-q(q-1) / 2]+G p$ \\
\hline
\end{tabular}

\subsection{Mixture of Multivariate-t Factor Analyzers}

The multivariate- $t$ analogue of the PGMMs, known as the mixture of multivariate $t$-factor analyzers (MMtFA) were introduced by Andrews and McNicholas (2011a,b).

In the MMtFAs, the component scale stucture is also parameterized as $\boldsymbol{\Sigma}_{g}=\boldsymbol{\Lambda}_{g} \boldsymbol{\Lambda}_{g}^{\prime}+$ $\boldsymbol{\Psi}_{g}$. By applying the constraints: $\boldsymbol{\Psi}_{g}=\psi_{g} \mathbf{I}, \boldsymbol{\Lambda}_{g}=\boldsymbol{\Lambda}$, and $\nu_{g}=\nu$, Andrews and McNicholas (2011a) created a family of six models, whose covariance parameters grow linearly with $p$, and Andrews and McNicholas (2011b) extended this to a family of 24 models. It is worth noting that the probabilistic principal $t$-component analyzer model, MPtCA, is a special case of the MMtFA model, where $\boldsymbol{\Psi}_{g}=\psi_{g} \mathbf{I}$. This family 
of 24 models is supported by the mmtfa package (Andrews et al., 2015).

\section{$2.9 \quad$ High-Dimensional Data Clustering}

Bouveyron et al. (2007) proposed a high-dimensional data clustering (HDDC) technique that is also based on an eigen-decomposition of the covariance structure of the Gaussian mixture model. This technique projects the observed data into a lowerdimensional subspace spanned by a subset of the eigenvectors of $\boldsymbol{\Sigma}_{g}$. Formally, given a data set $\left\{\mathbf{x}_{1}, \ldots, \mathbf{x}_{n}\right\}$ of $n$ data points in $\mathbb{R}^{p}$ with $G$ sub-populations, this method assumes that high-dimensional data mostly rests in lower-dimensional subspaces. This assumption can drastically reduce the number of covariance parameters that require estimation and result in an efficient parameter estimation scheme.

As with the GPCMs, Bouveyron et al. (2007) lets $\mathbf{D}_{g}$ be the orthogonal matrix of eigenvectors of $\boldsymbol{\Sigma}_{g}$, but instead considers a block-diagonal matrix, $\boldsymbol{\Delta}_{g}$, which contains the eigenvalues of $\boldsymbol{\Sigma}_{g}$. Formally, $\boldsymbol{\Delta}_{g}$ has the following form:

$$
\boldsymbol{\Delta}_{g}=\left(\begin{array}{ccc|ccc}
a_{1 g} & & 0 & & & \\
& \ddots & & & \mathbf{0} & \\
& & a_{d_{g} g} & & & \\
\hline & & & b_{g} & & 0 \\
& \mathbf{0} & & & \ddots & \\
& & & 0 & & b_{g}
\end{array}\right),
$$

where the upper left block is of size $d_{g} \times d_{g}, d_{g} \in\{1, p-1\}$ is the intrinsic dimension in each component, or cluster, and the lower right block is of size $\left(p-d_{g}\right) \times\left(p-d_{g}\right)$, 
with $a_{j g}>b_{g}$, for $j=1, \ldots, d_{g}$ and $g=1, \ldots, G$. Bouveyron et al. (2007) proposed two methods for estimating the intrinsic dimension in each component of this eigendecomposed GMM. The first approach utilizes the scree-test of Cattell (1966) and the second approaches utilizes the probabilistic Bayesian information Criterion (BIC; Schwarz, 1978), which is given by

$$
\mathrm{BIC}=2 l(\hat{\boldsymbol{\vartheta}})-\rho \log n,
$$

where $\rho$ is the number of free parameters in the model, $n$ is the number of observations, and $l(\hat{\boldsymbol{\vartheta}})$ is the maximized log-likelihood value. The eigenvectors associated with the eigenvalues $a_{j g}$, for $j=1, \ldots, d_{g}$, span a subspace $\mathbb{E}_{g} \in \mathbb{R}^{d_{g}}$ for each cluster, such that

$\boldsymbol{\mu}_{g} \in \mathbb{E}_{g}$. The affine subspace $\mathbb{E}_{g}^{\perp}$ is defined such that $\mathbb{E}_{g} \otimes \mathbb{E}_{g}^{\perp}=\mathbb{R}^{p}$ and $\boldsymbol{\mu}_{g} \in \mathbb{E}_{g}^{\perp}$. Each observation $\mathbf{x}_{i}$ is then projected onto the subspace $\mathbb{E}_{g}$, which is called the specific subspace of the $g$ th group, because most of the data are assumed to live on or near this subspace. This decomposition leads to 28 possible models by constraining the parameters $a_{j g}, b_{g}, \mathbf{D}_{g}, d_{g}$ across the $G$ components and $d_{g}$ dimensions. Of these 28 models, 14 have been implemented in the R package HDclassif (Berge et al., 2012). The fully unconstrained model will be referred to as UUUU and the letters C, G and D will denote completely constrained, constrained across groups and constrained across dimensions, respectively.

\subsection{Model Selection and Performance Assesment}

When fitting a family of mixture-models, it is necessary to choose the 'best' model. A very popular choice is the BIC, defined in Section 2.9. It is a widely used method and 
has been strongly established in theoretical and applied work (Leroux et al., 1992; Kass and Raftery, 1995; Kass and Wasserman, 1995; Keribin, 2000). It is important to note that the BIC can be used to approximate the Bayes factors (Kass and Raftery, 1995; Dasgupta and Raftery, 1998). In particular, Dasgupta and Raftery (1998) show that

$$
2 \log p\left(\mathbf{x} \mid \boldsymbol{\vartheta}_{g}\right) \approx \mathrm{BIC}_{G}
$$

where $p\left(\mathbf{x} \mid \boldsymbol{\vartheta}_{g}\right)$ is the integrated likelihood of the data using a $G$-component mixture model and $\mathrm{BIC}_{G}$ is the $\mathrm{BIC}$ value for the model with $G$ groups. The difference in $\mathrm{BIC}$ values for two models is an approximation to the Bayes factor, assuming that the prior distributions of the two models are equal.

For performance assessment, a usual choice is the adjusted Rand index (ARI; Hubert and Arabie, 1985). The ARI is used to assess class agreement between the true class labels and the predicted labels rendered by the clustering techniques. It was originally introduced to correct the Rand index (Rand, 1971), which is given by

$$
\mathrm{RI}=\frac{\text { number of pairwise agreements }}{\text { number of pairs }},
$$

where pairwise agreements refer to pairs of observations being correctly classified as coming from the same group and correctly classified as coming from different groups. The expected value of the Rand index is greater than 0 for a random classification, making it hard to interpret. Hence, the ARI has expected value equal to 0 , with a perfect classification being represented by a score of 1 . A negative ARI means that the classification performance is worse than would be expected under random classification. 


\section{Chapter 3}

\section{Methodology}

We now lay out some groundwork for a novel HDDC approach that utilizes mixtures of multivariate- $t$ distributions. This approach, which is the $t$-analouge of the HDDC models described in Section 2.9, rectifies a well-known shortcoming of the Gaussian mixture model-based approach to cluster analysis by incorporating a distribution that is robust to outliers. Like with all the clustering procedures introduced so far, our goal is to cluster a given data set $\left\{\mathbf{x}_{1}, \ldots, \mathbf{x}_{n}\right\}$ in $\mathbb{R}^{p}$ into $G$ homogeneous groups.

Herein, we use a mixture of multivariate- $t$ distributions, whose density is given by

$$
f\left(\mathbf{x} \mid \pi_{g}, \boldsymbol{\mu}_{g}, \boldsymbol{\Sigma}_{g}, \nu_{g}\right)=\sum_{g=1}^{G} \pi_{g} \frac{\Gamma\left[\left(\nu_{g}+p\right) / 2\right]\left|\boldsymbol{\Sigma}_{g}\right|^{-1 / 2}\left(\pi \nu_{g}\right)^{-p / 2}}{\Gamma\left[\nu_{g} / 2\right]\left[1+\delta\left(\mathbf{x}, \boldsymbol{\mu}_{g} \mid \boldsymbol{\Sigma}_{g}\right) / \nu_{g}\right]^{\left(\nu_{g}+p\right) / 2}},
$$

where all model parameters are defined for equations (2.2), (2.4) and (2.5).

The general multivariate- $t$ mixture model requires the estimation of the full covariance structure, so the number of parameters to estimate is $\mathcal{O}\left(p^{2}\right)$. As Bouveyron et al. (2007) describe, via the empty space phenomenon (Scott and Thompson, 1983), we can assume that most of the data live around lower-dimensional subspaces. By 
performing clustering in these lower-dimensional subspaces, the number of parameters to be estimated is reduced significantly.

\subsection{Formulating the $t$ HDDC Approach}

Analogous to the HDDC approach, we specify $\mathbf{D}_{g}$ to be the orthogonal matrix of eigenvectors and

$$
\Delta_{g}=\mathbf{D}_{g}^{\prime} \Sigma_{g} \mathbf{D}_{g}
$$

where $\boldsymbol{\Delta}_{g}$ is a class specific matrix of the form given in (2.10). Following Bouveyron et al. (2007) we define

$$
P_{g}(\mathbf{x})=\tilde{\mathbf{D}}_{g} \tilde{\mathbf{D}}_{g}^{\prime}\left(\mathbf{x}-\boldsymbol{\mu}_{g}\right)+\boldsymbol{\mu}_{g} \quad \text { and } \quad P_{g}^{\perp}(\mathbf{x})=\overline{\mathbf{D}}_{g} \overline{\mathbf{D}}_{g}^{\prime}\left(\mathbf{x}-\boldsymbol{\mu}_{g}\right)+\boldsymbol{\mu}_{g}
$$

as the projection of $\mathbf{x}$ on $\mathbb{E}_{g}$ and the projection of $\mathbf{x}$ on $\mathbb{E}_{g}^{\perp}$, respectively, where $\tilde{\mathbf{D}}_{g}$ consists of the first $d_{g}$ columns of $\mathbf{D}_{g}$, concatenated with $p-d_{g}$ zero columns, and $\overline{\mathbf{D}}_{g}=\mathbf{D}_{g}-\tilde{\mathbf{D}}_{g}$.

Formally, the covariance structure of each tHDDC mixture model has parameters $a_{j g}, b_{g}, \mathbf{D}_{g}, d_{g}, \nu_{g}$ for $j=1, \ldots, d_{g}$ and $g=1, \ldots, G$. It follows that applying groupwide constraints to the covariance parameters and allowing the degrees of freedom parameter, $\nu_{g}$, to either vary or be constrained across $G$ can lead to a total of 56 possible models. (The $t$ HDDC analogues of the HDDC models available in HDclassif are listed in Table 3.1). 


\subsection{Parameter Estimation}

In model-based clustering, the expectation-maximization (EM) algorithm (Dempster et al., 1977; McLachlan and Krishnan, 2008) is the usual choice when estimating the parameters of a finite mixture model. As the name suggests, the EM algorithm is an iterative procedure that alternates between two steps: an E-step and a M-step, until convergence is reached. On the E-step, the expected value of the complete-data loglikelihood is updated given the current parameter estimates. In the M-step, the same complete-data log-likelihood is maximized in terms of the model parameters. We use a variation of the EM algorithm called the expectation conditional-maximization (ECM) algorithm (Meng and Rubin, 1993), which replaces each M-step with multiple CM-steps. For each $t$ HDDC model the complete-data is made up of the observed $\mathbf{x}_{i}$, the latent $u_{i g}$, and the missing $z_{i g}$ for $i=1, \ldots, n$ and $g=1, \ldots, G$. The $z_{i g}$ are introduced to represent component membership. Formally, we write that

$$
z_{i g}= \begin{cases}1 & \text { if observation } \mathbf{x}_{i} \text { belongs to component } g \\ 0 & \text { otherwise. }\end{cases}
$$

The $u_{i g}$ is a realization of a gamma distributed random variable, $U_{i g}$, that arises because we exploit the fact that the multivariate- $t$ distribution is a normal-variance mixture as defined in Section 2.1. Formally, the random variable $U_{i g} \mid z_{i g}=1$ follows a $\mathcal{G}(\nu / 2, \nu / 2)$ distribution (Peel and McLachlan, 2000). Therefore, it will have density

$$
f(u ; \nu / 2, \nu / 2)=\frac{(\nu / 2)^{\nu / 2} u^{\nu / 2-1}}{\Gamma(\nu / 2)} \exp [(-\nu / 2) u]
$$

It follows that $U \mid \mathbf{X} \sim \mathcal{G}\left((\nu+p) / 2, \frac{\nu+\delta(\mathbf{x}, \boldsymbol{\mu} \mid \boldsymbol{\Sigma})}{2}\right)$. 


\subsubsection{The E-step}

For the general finite mixture model, the component indicator variables are usually replaced by their expected values,

$$
\mathbb{E}\left[Z_{i g} \mid \mathbf{x}_{i}\right]=\frac{\pi_{g} p_{g}\left(\mathbf{x} \mid \boldsymbol{\vartheta}_{g}\right)}{\sum_{h=1}^{G} \pi_{h} p_{h}\left(\mathbf{x} \mid \boldsymbol{\vartheta}_{h}\right)}=: \hat{z}_{i g}
$$

for $i=1, \ldots, n$ and $g=1, \ldots, G$. Unfortunately, this usually requires the computation of both the determinant and inverse of a $p \times p$ covariance matrix. To avoid these potentially cumbersome calculations, we follow Bouveyron et al. (2007) and derive a cost function that utilizes the projection functions: $P_{g}(x)$ and $P_{g}^{\perp}(x)$, defined in Section 3.1. The derivation of the cost function is as follows: first, note that we can write

$$
\begin{aligned}
& -2 \log f_{t}(\mathbf{x} \mid \boldsymbol{\mu}, \boldsymbol{\Sigma}, \nu)=-2 \log \Gamma[(\nu+p) / 2]+2 \log \Gamma[\nu / 2]+p(\log \nu+\log \pi) \\
& +(\nu+p) \log \left[1+\frac{1}{\nu}\left(\|\boldsymbol{\mu}-P(\mathbf{x})\|_{\mathbf{A}}^{2}+\frac{1}{b}\left\|\mathbf{x}-P^{\prime}(\mathbf{x})\right\|^{2}\right)\right]+\sum_{j=1}^{d} \log a_{j}+(p-d) \log b,
\end{aligned}
$$

where $\|\mathbf{x}\|_{\mathbf{A}}^{2}=\mathbf{x} \mathbf{A} \mathbf{x}^{\prime}$ with $\mathbf{A}=\tilde{\mathbf{D}}_{g} \boldsymbol{\Delta}_{g} \tilde{\mathbf{D}}_{g}^{\prime}$, and all other values are as previously defined. So, on the E-step of the proposed ECM algorithm we replace each $z_{i g}$ with

$$
\hat{z}_{i g}=\frac{1}{\sum_{h=1}^{G} \exp \left[\frac{1}{2}\left(K_{g}\left(\mathbf{x}_{i}\right)-K_{h}\left(\mathbf{x}_{i}\right)\right)\right.},
$$


where we refer to

$$
\begin{aligned}
& K_{g}\left(\mathbf{x}_{i}\right)=-2 \log \Gamma\left[\left(\nu_{g}+p\right) / 2\right]+2 \log \Gamma\left[\nu_{g} / 2\right]+\left(p-d_{g}\right) \log b_{g}+p\left(\log \nu_{g}+\log \pi\right) \\
& +\left(\nu_{g}+p\right) \log \left[1+\frac{1}{\nu_{g}}\left(\left\|\boldsymbol{\mu}_{g}-P_{g}(\mathbf{x})\right\|_{\mathbf{A}_{g}}^{2}+\frac{1}{b_{g}}\left\|\mathbf{x}-P_{g}^{\prime}(\mathbf{x})\right\|^{2}\right)\right]+\sum_{j=1}^{d_{g}} \log a_{j g}-2 \log \pi_{g},
\end{aligned}
$$

for $i=1, \ldots, n$ and $g=1, \ldots, G$, as the cost function. Each $u_{i g}$ is then replaced by their expected values

$$
\mathbb{E}\left[U_{i g} \mid \mathbf{x}_{i}, z_{i g}=1\right]=\frac{\nu_{g}+p}{\nu_{g}+\left\|\boldsymbol{\mu}_{g}-P\left(\mathbf{x}_{i}\right)\right\|_{\mathbf{A}}^{2}+\frac{1}{b_{g}}\left\|\mathbf{x}-P^{\prime}\left(\mathbf{x}_{i}\right)\right\|^{2}}=: \hat{u}_{i g},
$$

for $i=1, \ldots, n$ and $g=1, \ldots, G$ (cf. Peel and McLachlan, 2000; Andrews and McNicholas, 2012). In this update we exploit a part of the cost function derivation (given in Appendix A), which states that

$$
\delta\left(\mathbf{x}, \boldsymbol{\mu}_{g} \mid \boldsymbol{\Sigma}_{g}\right)=\left\|\boldsymbol{\mu}_{g}-P\left(\mathbf{x}_{i}\right)\right\|_{\mathbf{A}}^{2}+\frac{1}{b_{g}}\left\|\mathbf{x}-P^{\prime}\left(\mathbf{x}_{i}\right)\right\|^{2} .
$$

\subsubsection{The CM-steps}

On the first CM-step we update the mixing proportions and component location parameter using

$$
\hat{\pi}_{g}=\frac{n_{g}}{n} \quad \text { and } \quad \hat{\boldsymbol{\mu}}_{g}=\frac{\sum_{i=1}^{n} \hat{z}_{i g} \hat{u}_{i g} \mathbf{x}_{i}}{\sum_{i=1}^{n} \hat{z}_{i g} \hat{u}_{i g}}
$$

respectively, where $n_{g}=\sum_{i=1}^{n} \hat{z}_{i g}$. The degrees of freedom parameter, $\nu_{g}$, is updated using the closed form approximation given in Andrews et al. (2017). Formally, we let

$$
\hat{\nu}_{g} \approx \frac{-\exp (k)+2 \exp \left(\varphi\left(\frac{\hat{\nu}_{g}^{\text {old }}}{2}\right)+\frac{1-\hat{\nu}_{g}^{\text {old }}}{2}\right) \exp (k)}{1-\exp (k)}
$$


with

$$
k=-1-\frac{1}{n_{g}} \sum_{g=1}^{G} \sum_{i=1}^{n} \hat{z}_{i g}\left(\log \hat{u}_{i g}-\hat{u}_{i g}\right)-\varphi\left(\frac{\hat{\nu}_{g}^{\text {old }}+p}{2}\right)+\log \left(\frac{\hat{\nu}_{g}^{\text {old }}+p}{2}\right)
$$

where $\hat{\nu}_{g}^{\text {old }}$ is the estimate of $\nu_{g}$ from the previous iteration of this ECM algorithm, and $\varphi(\cdot)$ is the digamma function.

For each tHDDC model, the updates on the second CM step are analogous to those given in Bouveyron et al. (2007). For illustrative purposes, we outline how to update each covariance parameter for the UUUUU model, i.e., the model where $a_{j g}, b_{g}, \mathbf{D}_{g}, d_{g}, \nu_{g}$ are free to vary across all $g=1, \ldots, G$ and $j=1, \ldots, d_{g}$.

First, we calculate the intrinsic dimension, $d_{g}$. For each value of $j \in\{1, p-1\}$ we compute

$$
\begin{aligned}
l(\hat{\boldsymbol{\vartheta}})=-\frac{n}{2}\left(d_{j} \log a_{j g}+\left(p-d_{j}\right) \log b_{g}-2 \log \pi_{g}+\log \nu_{g}\right. \\
\left.+\log \pi-2 \log \Gamma\left[\left(\nu_{g}+p\right) / 2\right]+2 \log \Gamma\left(\nu_{g} / 2\right)\right)
\end{aligned}
$$

and set $d_{g}$ equal to the value of $d_{j}$ that maximizes the BIC values associated with the $\log$-likelihood values found using (3.6). Then we let $\mathbf{D}_{g}$ be the eigenvectors of $\hat{\boldsymbol{\Sigma}}_{g}$, where

$$
\hat{\boldsymbol{\Sigma}}_{g}=\frac{1}{n_{g}} \sum_{i=1}^{n} \hat{z}_{i g} \hat{u}_{i g}\left(\mathbf{x}_{i}-\hat{\boldsymbol{\mu}}_{g}\right)\left(\mathbf{x}_{i}-\hat{\boldsymbol{\mu}}_{g}\right)^{\prime} .
$$

Each $a_{j g}$, for $j=1, \ldots, d_{g}$ and $g=1, \ldots, G$ is then replaced with the first $d_{g}$ eigenvalues of $\hat{\boldsymbol{\Sigma}}_{g}$ and we estimate $b_{g}$ using

$$
\hat{b}_{g}=\frac{1}{\left(p-d_{g}\right)}\left(\operatorname{tr}\left(\hat{\boldsymbol{\Sigma}}_{g}\right)-\sum_{j=1}^{d_{g}} a_{j g}\right) .
$$




\subsection{Convergence}

For the proposed ECM algorithm, we initialize each model using either k-means clustering or random starting values and use the Aitkin's acceleration (Aitken, 1926) procedure to determine if the algorithm has converged. That is, we consider this ECM algorithm to have converged when $l_{\infty}^{(k+1)}-l^{(k)}<\epsilon$, where $\epsilon=10^{-2}$ (see Lindsay, 1995; McNicholas et al., 2010). In this criterion, $l^{(k)}$ is the log-likelihood value at iteration $(k)$ and $l_{\infty}^{(k+1)}$ is the asymptotic estimate of the log-likelihood at iteration $(k+1)$. Formally,

$$
l_{\infty}^{(k+1)}=l^{(k)}+\frac{1}{1-a^{(k)}}\left(l^{(k+1)}-l^{(k)}\right),
$$

where

$$
a^{(k)}=\frac{l^{(k+1)}-l^{(k)}}{l^{(k)}-l^{(k-1)}} .
$$


Table 3.1: Nomenclature, covariance decomposition and number of free covariance parameters for the $t$ HDDC models. For constaints on $a_{j g}$, U represents unconstrained, $\mathrm{D}$ represents constrained accross dimension, $\mathrm{G}$ represents constrained across groups and $\mathrm{C}$ represents constrained accross both dimension and group. For all other components, $\mathrm{U}$ and $\mathrm{C}$ are unconstrained and constrained across groups, respectively. For the number of free parameters, $\rho=G p+G+1$ is the number of parameters required to estimate the mean and proportions. The number of parameters required to estimate $\tilde{D}_{g}, \tilde{D}$ and are $\bar{\tau}=d_{g}\left[p-\left(d_{g}+1\right) / 2\right]$ and $\tau=d[p-(d+1) / 2]$, and $s=\sum_{g=1}^{G} d_{g}$.

\begin{tabular}{|c|c|c|c|c|c|c|}
\hline Model & $a_{j g}=a_{g} / a_{j}$ & $b_{g}=b$ & $\mathbf{D}_{g}=\mathbf{D}$ & $d_{g}=d$ & $\nu_{g}=\nu$ & $\begin{array}{l}\text { Number of } \\
\text { Cov. Parameters }\end{array}$ \\
\hline UUUUU & $\mathrm{U}$ & $\mathrm{U}$ & $\mathrm{U}$ & $\mathrm{U}$ & $\mathrm{U}$ & $\rho+\bar{\tau}+3 G+s$ \\
\hline UCUUU & $\mathrm{U}$ & $\mathrm{C}$ & $\mathrm{U}$ & $\mathrm{U}$ & $\mathrm{U}$ & $\rho+\bar{\tau}+2 G+s+1$ \\
\hline DUUUU & $\mathrm{D}$ & $\mathrm{U}$ & $\mathrm{U}$ & $\mathrm{U}$ & $\mathrm{U}$ & $\rho+\bar{\tau}+4 G$ \\
\hline CUUUU & $\mathrm{C}$ & $\mathrm{U}$ & $\mathrm{U}$ & $\mathrm{U}$ & $\mathrm{U}$ & $\rho+\bar{\tau}+3 G+1$ \\
\hline DCUUU & $\mathrm{D}$ & $\mathrm{C}$ & $\mathrm{U}$ & $\mathrm{U}$ & $\mathrm{U}$ & $\rho+\bar{\tau}+3 G+1$ \\
\hline CCUUU & $\mathrm{C}$ & $\mathrm{C}$ & $\mathrm{U}$ & $\mathrm{U}$ & $\mathrm{U}$ & $\rho+\bar{\tau}+2 G+2$ \\
\hline UUUCU & $\mathrm{U}$ & $\mathrm{U}$ & $\mathrm{U}$ & $\mathrm{C}$ & $\mathrm{U}$ & $\rho+G(\tau+d+2)+1$ \\
\hline UCUCU & $\mathrm{U}$ & $\mathrm{C}$ & $\mathrm{U}$ & $\mathrm{C}$ & $\mathrm{U}$ & $\rho+G(\tau+d+1)+2$ \\
\hline DUUCU & $\mathrm{D}$ & $\mathrm{U}$ & $\mathrm{U}$ & $\mathrm{C}$ & $\mathrm{U}$ & $\rho+G(\tau+2+1)+1$ \\
\hline CUUCU & $\mathrm{C}$ & $\mathrm{U}$ & $\mathrm{U}$ & $\mathrm{C}$ & $\mathrm{U}$ & $\rho+G(\tau+2)+2$ \\
\hline DCUCU & $\mathrm{D}$ & $\mathrm{C}$ & $\mathrm{U}$ & $\mathrm{C}$ & $\mathrm{U}$ & $\rho+G(\tau+2)+2$ \\
\hline CCUCU & $\mathrm{C}$ & $\mathrm{C}$ & $\mathrm{U}$ & $\mathrm{C}$ & $\mathrm{U}$ & $\rho+G(\tau+1)+3$ \\
\hline GCCCU & G & $\mathrm{C}$ & $\mathrm{C}$ & $\mathrm{C}$ & $\mathrm{U}$ & $\rho+\tau+d+G+2$ \\
\hline CCCCU & $\mathrm{C}$ & $\mathrm{C}$ & $\mathrm{C}$ & $\mathrm{C}$ & $\mathrm{U}$ & $\rho+\tau+G+3$ \\
\hline UUUUC & $\mathrm{U}$ & $\mathrm{U}$ & $\mathrm{U}$ & $\mathrm{U}$ & $\mathrm{C}$ & $\rho+\bar{\tau}+2 G+s+1$ \\
\hline UCUUC & $\mathrm{U}$ & $\mathrm{C}$ & $\mathrm{U}$ & $\mathrm{U}$ & $\mathrm{C}$ & $\rho+\bar{\tau}+G+s+2$ \\
\hline DUUUC & $\mathrm{D}$ & $\mathrm{U}$ & $\mathrm{U}$ & $\mathrm{U}$ & $\mathrm{C}$ & $\rho+\bar{\tau}+3 G+1$ \\
\hline CUUUC & $\mathrm{C}$ & $\mathrm{U}$ & $\mathrm{U}$ & $\mathrm{U}$ & $\mathrm{C}$ & $\rho+\bar{\tau}+2 G+2$ \\
\hline DCUUC & $\mathrm{D}$ & $\mathrm{C}$ & $\mathrm{U}$ & $\mathrm{U}$ & $\mathrm{C}$ & $\rho+\bar{\tau}+2 G+2$ \\
\hline CCUUC & $\mathrm{C}$ & $\mathrm{C}$ & $\mathrm{U}$ & $\mathrm{U}$ & $\mathrm{C}$ & $\rho+\bar{\tau}+G+3$ \\
\hline UUUCC & $\mathrm{U}$ & $\mathrm{U}$ & $\mathrm{U}$ & $\mathrm{C}$ & $\mathrm{C}$ & $\rho+G(\tau+d+1)+2$ \\
\hline UCUCC & $\mathrm{U}$ & $\mathrm{C}$ & $\mathrm{U}$ & $\mathrm{C}$ & $\mathrm{C}$ & $\rho+G(\tau+d)+3$ \\
\hline DUUCC & $\mathrm{D}$ & $\mathrm{U}$ & $\mathrm{U}$ & $\mathrm{C}$ & $\mathrm{C}$ & $\rho+G(\tau+2)+2$ \\
\hline CUUCC & $\mathrm{C}$ & $\mathrm{U}$ & $\mathrm{U}$ & $\mathrm{C}$ & $\mathrm{C}$ & $\rho+G(\tau+1)+3$ \\
\hline DCUCC & $\mathrm{D}$ & $\mathrm{C}$ & $\mathrm{U}$ & $\mathrm{C}$ & $\mathrm{C}$ & $\rho+G(\tau+1)+3$ \\
\hline CCUCC & $\mathrm{C}$ & $\mathrm{C}$ & $\mathrm{U}$ & $\mathrm{C}$ & $\mathrm{C}$ & $\rho+G \tau+4$ \\
\hline GCCCC & G & $\mathrm{C}$ & $\mathrm{C}$ & $\mathrm{C}$ & $\mathrm{C}$ & $\rho+\tau+d+3$ \\
\hline $\mathrm{CCCCC}$ & $\mathrm{C}$ & $\mathrm{C}$ & $\mathrm{C}$ & $\mathrm{C}$ & $\mathrm{C}$ & $\rho+\tau+4$ \\
\hline
\end{tabular}




\section{Chapter 4}

\section{Application}

\subsection{Computational Considerations}

The data analyses will be treated as genuine clustering problems, where the true classifications are not known. Because we do have the true class labels, the ARI will be used to assess class agreement between the true and predicted class labels. In all applications, the best fitting models will be chosen using the BIC.

All analyses are performed in $\mathrm{R}$ version 3.3.2 (R Core Team, 2016) for Linux 6.5 ${ }^{1}$. Both the HDDC and $t$ HDDC models are fit with $G=1, \ldots, 10$. It is important to note that the only HDDC models considered are ones with a monotonic likelihood.

\footnotetext{
${ }^{1}$ Using a 32-core Intel Xeon E5 server with 256GB RAM running 64-bit CentOS
} 


\subsection{Simulation Studies}

We use a simulation study to highlight the aforementioned drawback of the considered mixture of multivariate Gaussian distributions. Ten data sets were simulated from a two-component multivariate- $t$ distribution with $\nu_{1}=2$ and $\nu_{2}=3$. Figure 4.1 provides an illustration of the first three dimensions of one of the simulated data sets. In each component, observations are scattered from the mean, with many outliers on far ends of the clusters. Table 4.1 gives the classification results for the tHDDC and HDDC models when fitted to the simulated data set. As expected, the tHDDC approach outperforms the HDDC approach, achieving a near perfect classification. The relatively small standard deviation reveals that the selected $t$ HDDC models are
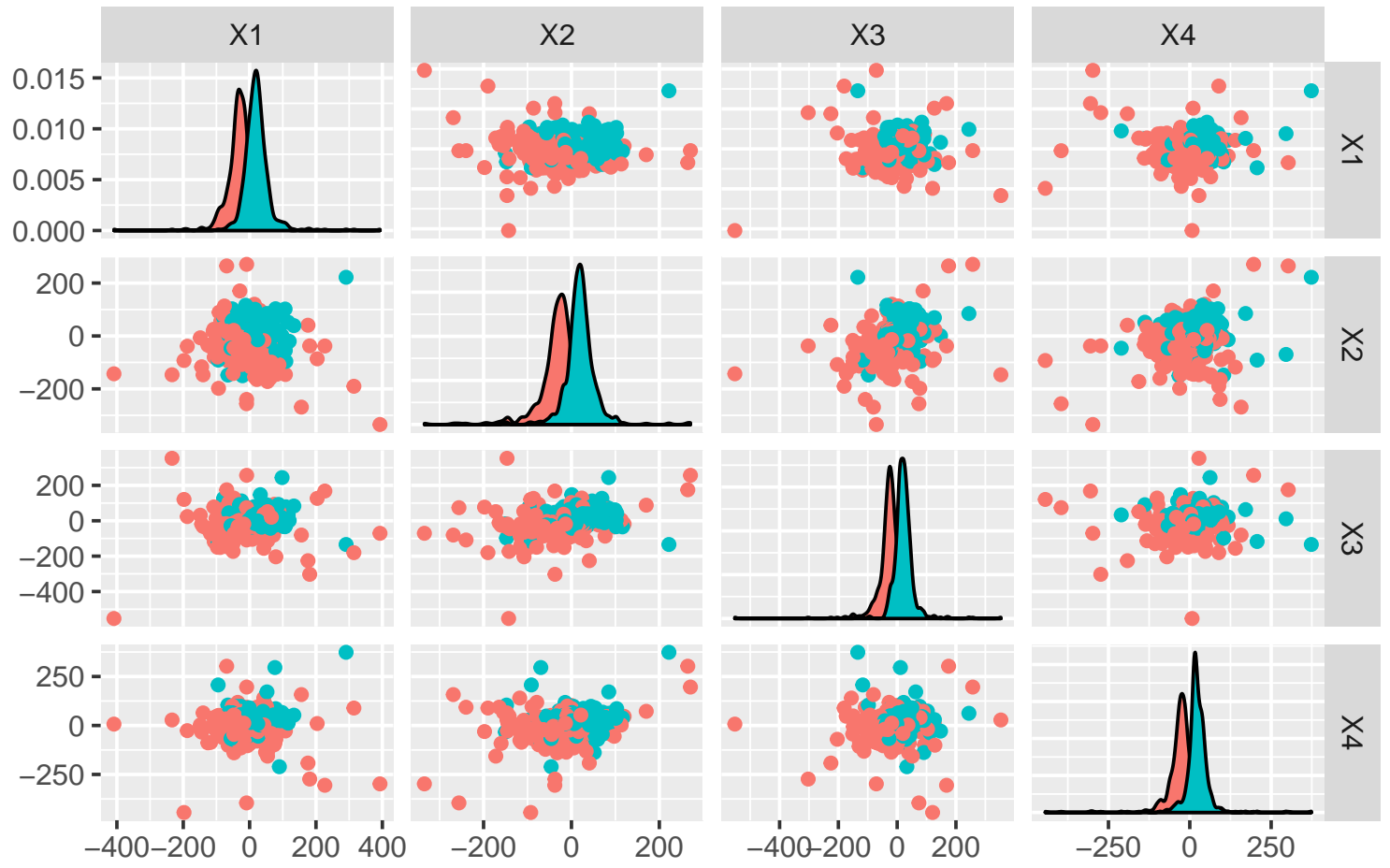

Figure 4.1: Pairs and density plots of the first three dimensions of one multivariate- $t$ simulated data set, coloured by true groups. 
consistently returning an exceptional classification performance, whereas the selected HDDC models are using extra components to account for the increased variation in the simulated data sets.

Table 4.1: Mean and standard deviation of ARI values returned by the best fitting HDDC and $t$ HDDC models found by the BIC for the simulated multivariate- $t$ data sets.

\begin{tabular}{lcc}
\hline & Mean of ARI & Standard Deviation of ARI \\
\hline HDDC & 0.021 & 0.012 \\
$t$ HDDC & 0.995 & 0.005 \\
\hline
\end{tabular}

\subsection{Fisher's Irises}

In our first real data analysis, we consider Fisher's famous iris data set, which is available in the R package datasets and gclus (Hurley, 2012). It is composed of four explanatory variables: sepal length, sepal width, petal length, and petal width, measured in centimetres. There are three species of the plant: Setosa, Versicolour and Virginica. Table 4.2 gives the classification results.

Both the best fitting HDDC and tHDDC models return a very good classification of the irises, with the best fitting tHDDC model outperforming the corresponding HDDC model. Across the three selected components, both the best fitting HDDC and $t$ HDDC models use a varying number of eigenvalues and eigenvectors with a

Table 4.2: Model decomposition, number of components, BIC and ARI values for the best fitting tHDDC and HDDC models when fitted for $G=1, \ldots, 10$ components for the Fisher's irises.

\begin{tabular}{llrrr}
\hline & Model & $G$ & BIC & ARI \\
\hline HDDC & UUUC & 3 & -588.01 & 0.868 \\
$t$ HDDC & UUUCC & 3 & -646.33 & 0.904 \\
\hline
\end{tabular}


common intrinsic dimension. Specifically, the selected $t$ HDDC models uses a common dimension of one and constrained degrees of freedom. In total, this model misclassifies only 5 irises (see Table 4.3).

Figure 4.2 shows the data in the one dimensional subspace for all three groups. The clusters found by the selected $t$ HDDC model are clear and well separated.

Table 4.3: A classification table showing the results for the selected three-component UUUCC $t$ HDDC model for the iris data.

\begin{tabular}{lccc}
\hline & A & B & C \\
\hline Setosa & 50 & 0 & 0 \\
Versicolor & 0 & 45 & 5 \\
Virginica & 0 & 0 & 50 \\
\hline
\end{tabular}
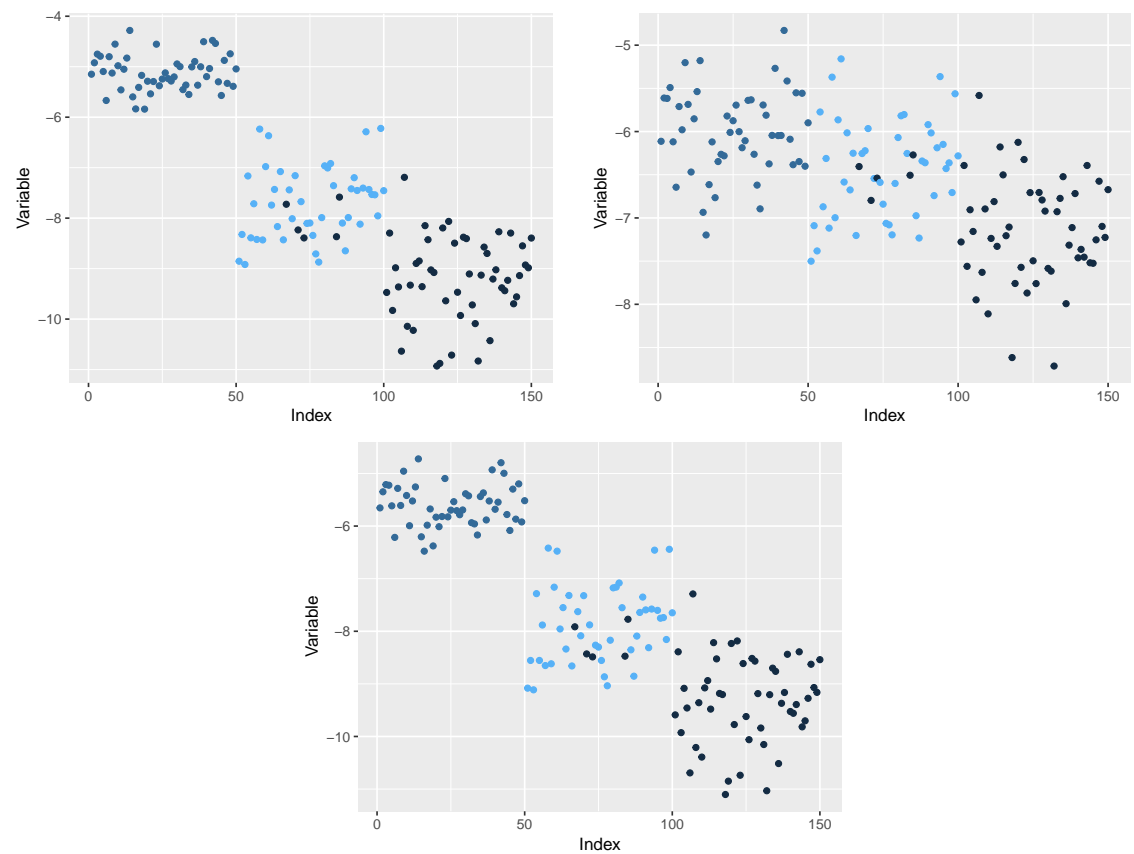

Figure 4.2: The iris data coloured by the classes found by the best fitting $t$ HDDC model and projected onto the one-dimensional spaces for each component. 


\subsection{Italian Wines}

The Italian wines data set, available as wine in the R package pgmm (McNicholas et al., 2015), is composed of 178 Italian wines on which 27 measurements are taken (Forina et al., 1986). The wines come from three different cultivars and are classified based on which one they come from: Barolo, Grignolino and Barbera. The $t$ HDDC models are fit using $G=1, \ldots, 5$, as the BIC will select a four-component mixture model. Table 4.4 gives the classification results. Note that when fitting only three-component HDDC and tHDDC models, the selected GCCC and GCCCC models gave the same classification result $(\mathrm{ARI}=0.933)$.

We can see that the best fitting HDDC model overfits by selecting a model with eight components. Although the best $t$ HDDC model did not have three groups, the four group solution gives a superior classification performance (see Table 4.4). The model selected by the BIC was the GCCCC model, with a common intrinsic dimension of 4. Looking at Figure 4.3, in the four dimensional subspace, we can see that there are no clear and well separated clusters.

Table 4.4: Model decomposition, number of components, BIC and ARI values for the best fitting HDDC and $t$ HDDC models when fitted for $G=1, \ldots, 10$ components for the Italian wines data.

\begin{tabular}{llrrr}
\hline & Model & $G$ & BIC & ARI \\
\hline HDDC & GCCC & 8 & $-12,071.63$ & 0.658 \\
$t$ HDDC & GCCCC & 4 & $-11,965.26$ & 0.758 \\
\hline
\end{tabular}



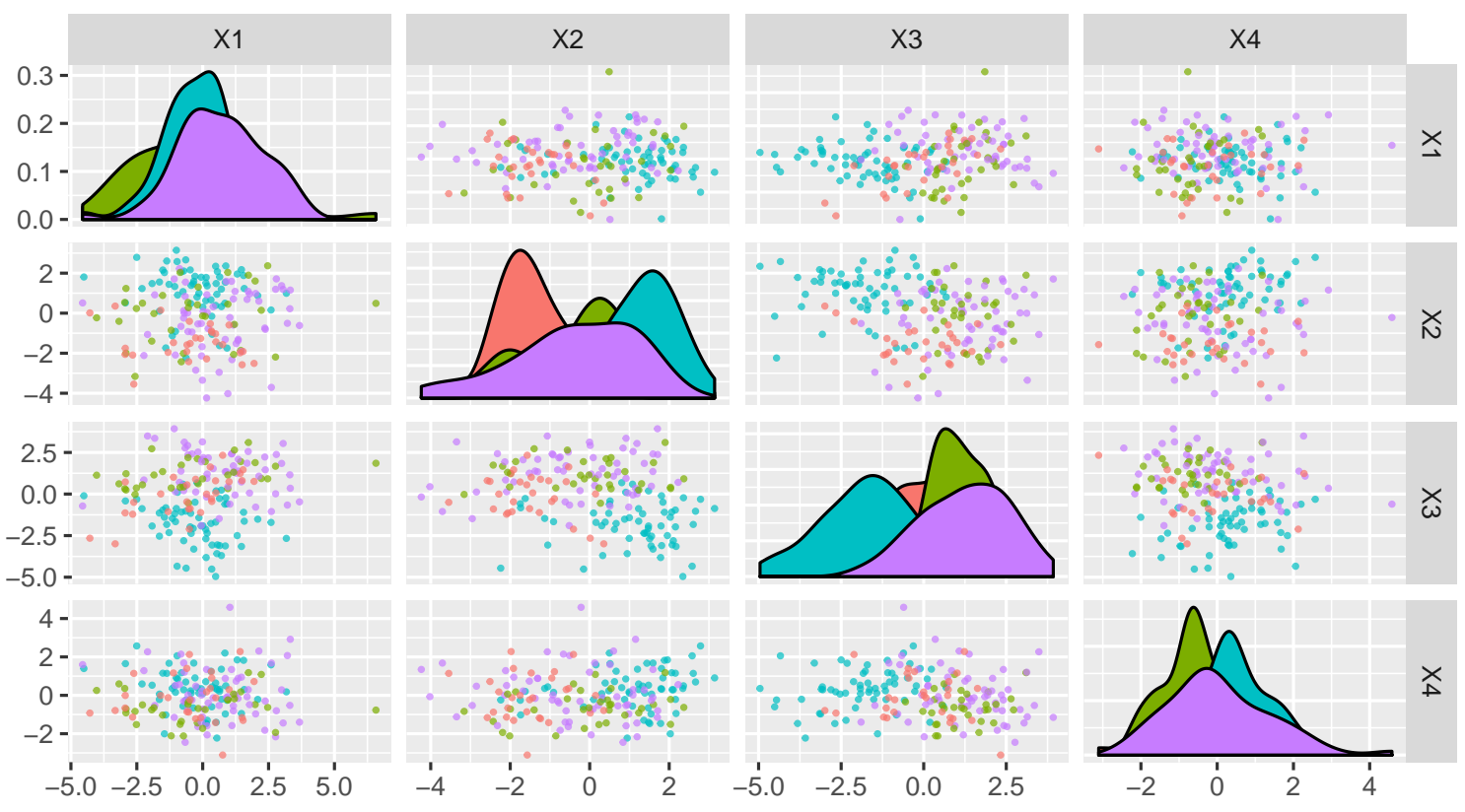

Figure 4.3: Pairs and density plots of the Italian wines data colored by classes found by the best fitting $t$ HDDC model and projected onto the four-dimensional subspace.

\subsection{Martian Surface}

This data set was retrieved by the OMEGA instrument (Mars Express, ESA; Bibring et al., 2004). The OMEGA instrument is used for characterization of the Martian surface based on physical and chemical composition. This can include classes of silicates, hydrated minerals, ices and more. The data used is based on one $300 \times 128$ raw image. It contains 255 variables on 38,400 observations. With a physical model, eight groups were found and for the purpose of this analysis, these will be treated as true groups; however, the best determination of model performance here is based on efficacy for image reconstruction. For $G=8$ components, $t$ HDDC does a little better than HDDC; however, neither performs well (Table 4.5). 
Although the physical model suggests eight groups, experts in the field are interested in exploring a five group solution (Bouveyron et al., 2007). Both HDDC and $t$ HDDC models are applied to this data with $G=5$, and the selected $t$ HDDC model recovers the clusters better than the selected HDDC model (see Table 4.6).

In Table 4.7, we can see that the classification results returned by the selected $t$ HDDC and HDDC models are quite different. Furthermore, comparing the recovered image based on the predicted classes to the original image (see Figure 4.4), the utility of the model becomes clear, i.e., the physical details are generally recovered very well.

Table 4.5: Model decomposition, BIC and ARI values for the selected eightcomponent HDDC and $t$ HDDC models for the Martian surface data.

\begin{tabular}{lllrr}
\hline & $G$ & Model & BIC & ARI \\
\hline HDDC & 8 & CUUU & $62,460,591$ & 0.319 \\
$t$ HDDC & 8 & CCCCC & $64,249,591$ & 0.351 \\
\hline
\end{tabular}

Table 4.6: Model decomposition, BIC and ARI values for the selected five-component HDDC and $t$ HDDC models for the Martian surface data.

\begin{tabular}{lllrr}
\hline & $G$ & Best Model & BIC & ARI \\
\hline HDDC & 5 & UUUU & $61,956,344$ & 0.472 \\
$t$ HDDC & 5 & UCUUC & $70,120,085$ & 0.645 \\
\hline
\end{tabular}

Table 4.7: A classification table comparing the best fitting five-component $t$ HDDC and HDDC models for the Martian surface data.

\begin{tabular}{ccccccc}
\hline & & \multicolumn{5}{c}{ HDDC } \\
\cline { 3 - 7 } & & $\mathrm{A}$ & $\mathrm{B}$ & $\mathrm{C}$ & $\mathrm{D}$ & $\mathrm{E}$ \\
\hline \multirow{4}{*}{$t$ HDDC } & 1 & 10744 & 22 & 0 & 0 & 2973 \\
& 2 & 1019 & 1598 & 0 & 99 & 785 \\
& 3 & 0 & 39 & 7807 & 4372 & 4 \\
& 4 & 2 & 319 & 1 & 4871 & 716 \\
& 5 & 605 & 914 & 4 & 283 & 1223 \\
\hline
\end{tabular}



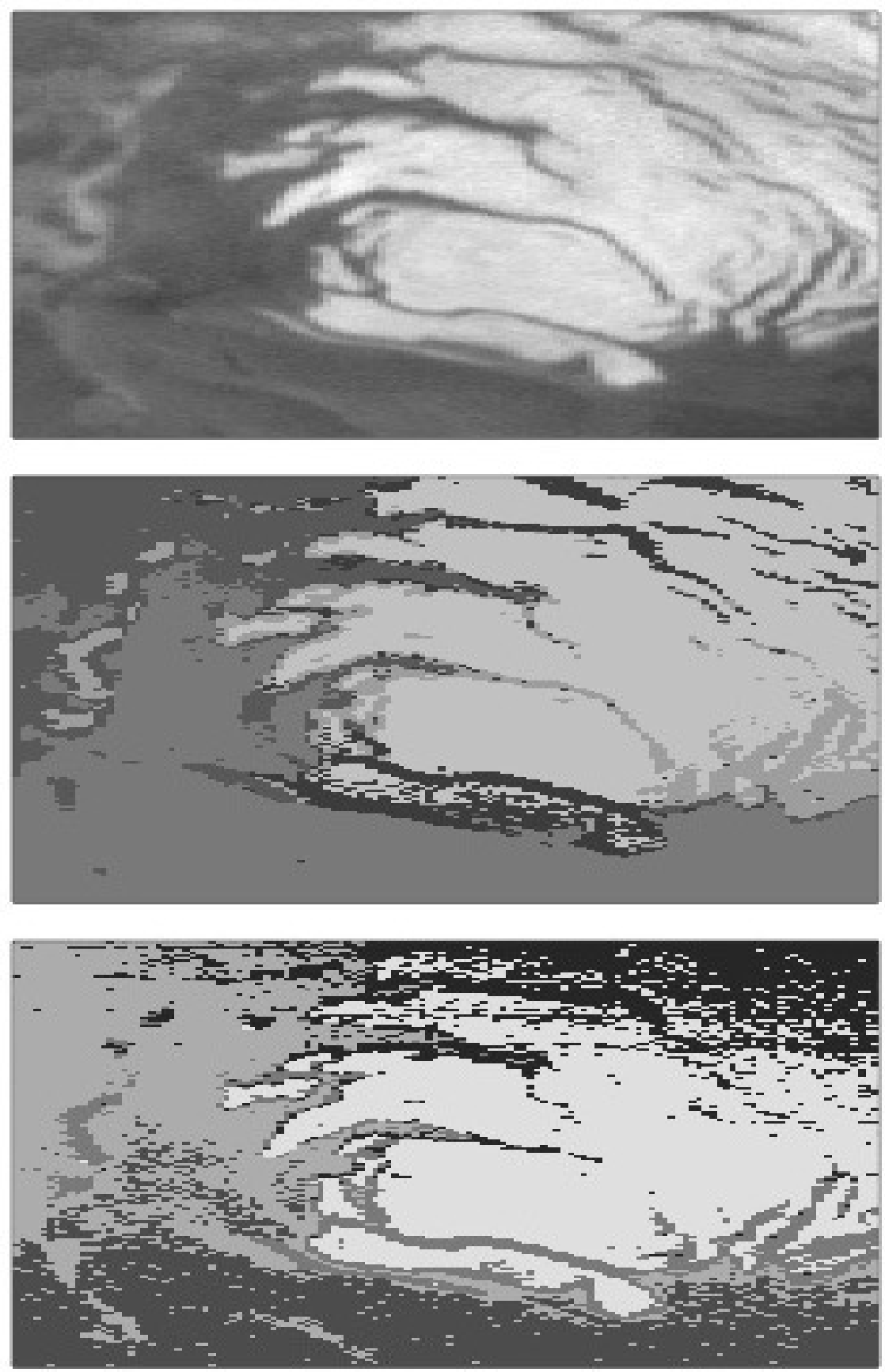

Figure 4.4: Three images of the Martian surface constructed using spectral data collected by the OMEGA instrument (top), the parameter estimates of the best-fitting $t$ HDDC model (middle) and the parameter estimates of the best-fitting HDDC model (bottom). 


\section{Chapter 5}

\section{Conclusions and Future Work}

A new family of multivariate- $t$ mixture models has been proposed. The $t$ HDDC approach is an extension of the HDDC approach that incorporates the multivariate- $t$ distribution, allowing for a more robust clustering scheme. A total of 28 models have been developed and the need for these models was shown through a simulation study which demonstrated their flexibility in recognizing outliers. The models were tested on both simulated and real data sets and show superior results when compared to the HDDC family. In particular, the results on the high-dimensional Martian surface data show that image recovery can be greatly improved. Overall, the added degrees of freedom parameter allows for more flexible clusters and a more flexible modelling structure than HDDC.

A further direction to this research would be to explore different model and dimension selection criteria. Some include the integrated completed likelihood criterion (Biernacki et al., 2000) and the approximate weight of evidence (Banfield and Raftery, 1993). In addition, this method can be extended to include skewed mixture-models. Examples include the mixture of multivariate skew- $t$ distributions (Lin, 2010; Murray 
et al., 2014, 2017), the mixture of shifted asymmetric Laplace distributions (Franczak et al., 2014), the mixture of variance-gamma distributions (McNicholas et al., 2017), and the mixture of generalized hyperbolic distributions (Browne and McNicholas, 2015). 


\section{Appendix A}

\section{Cost Function Derivations}

The cost function for a mixture of multivariate- $t$ distributions can be derived as follows:

$$
\begin{aligned}
& \log \xi_{p}(\mathbf{x} \mid \boldsymbol{\mu}, \boldsymbol{\Sigma}, \nu)=\log \left[\frac{\Gamma[(\nu+p) / 2]|\boldsymbol{\Sigma}|^{-1 / 2}(\nu \pi)^{-p / 2}}{\Gamma[\nu / 2][1+\delta(\mathbf{x}, \boldsymbol{\mu} \mid \boldsymbol{\Sigma}) / \nu]^{(\nu+p) / 2}}\right] \\
& \log \xi_{p}(\mathbf{x} \mid \boldsymbol{\mu}, \boldsymbol{\Sigma}, \nu)=\log \Gamma[(\nu+p) / 2]-\log \left[|\boldsymbol{\Sigma}|^{1 / 2}\right]-\log \left[(\nu \pi)^{p / 2}\right]-\log \Gamma[\nu / 2]-\log \left[\left(1+\frac{1}{\nu} \delta(\mathbf{x}, \boldsymbol{\mu} \mid \boldsymbol{\Sigma})\right)^{(\nu+p) / 2}\right], \\
& \log \xi_{p}(\mathbf{x} \mid \boldsymbol{\mu}, \boldsymbol{\Sigma}, \nu)=\log \Gamma[(\nu+p) / 2]-\frac{p}{2} \log [\nu \pi]-\log \Gamma[\nu / 2]-\frac{1}{2} \log |\boldsymbol{\Sigma}| \\
&-\frac{(\nu+p)}{2} \log \left[1+\frac{1}{\nu} \delta(\mathbf{x}, \boldsymbol{\mu} \mid \boldsymbol{\Sigma})\right], \\
&-2 \log \xi_{p}(\mathbf{x} \mid \boldsymbol{\mu}, \boldsymbol{\Sigma}, \nu)=-2 \log \Gamma[(\nu+p) / 2]+2 \log \Gamma[\nu / 2]+p(\log \nu+\log \pi) \\
&+\log \left|\left(\mathbf{D} \boldsymbol{\Delta} \mathbf{D}^{\prime}\right)\right|+(\nu+p) \log \left[1+\frac{1}{\nu}(\mathbf{x}-\boldsymbol{\mu})^{\prime}\left(\mathbf{D} \boldsymbol{\Delta} \mathbf{D}^{\prime}\right)^{-1}(\mathbf{x}-\boldsymbol{\mu})\right]
\end{aligned}
$$




$$
\begin{aligned}
-2 \log \xi_{p}(\mathbf{x} \mid \boldsymbol{\mu}, \boldsymbol{\Sigma}, \nu)= & -2 \log \Gamma[(\nu+p) / 2]+2 \log \Gamma[\nu / 2]+p(\log \nu+\log \pi)+\log |\boldsymbol{\Delta}| \\
& +(\nu+p) \log \left[1+\frac{1}{\nu}\left(\left\|\tilde{\mathbf{D}} \tilde{\mathbf{D}}^{\prime}(\mathbf{x}-\boldsymbol{\mu})\right\|_{\mathbf{A}}^{2}+\frac{1}{b}\left\|\overline{\mathbf{D}} \overline{\mathbf{D}}^{\prime}(\mathbf{x}-\boldsymbol{\mu})\right\|^{2}\right)\right], \\
-2 \log \xi_{p}(\mathbf{x} \mid \boldsymbol{\mu}, \boldsymbol{\Sigma}, \nu)= & -2 \log \Gamma[(\nu+p) / 2]+2 \log \Gamma[\nu / 2]+p(\log \nu+\log \pi) \\
+ & (\nu+p) \log \left[1+\frac{1}{\nu}\left(\|\boldsymbol{\mu}-P(\mathbf{x})\|_{\mathbf{A}}^{2}+\frac{1}{b}\left\|\mathbf{x}-P^{\prime}(\mathbf{x})\right\|^{2}\right)\right] \\
+ & \sum_{j=1}^{d} \log a_{j g}+(p-d) \log b .
\end{aligned}
$$

Therefore, we can write

$$
z_{i g}=\frac{1}{\sum_{h=1}^{G} \exp \left\{\frac{1}{2}\left(K_{g}\left(\mathbf{x}_{i}\right)-K_{h}\left(\mathbf{x}_{i}\right)\right)\right\}},
$$

where

$$
\begin{aligned}
K_{g}(\mathbf{x})= & -2 \log \Gamma\left[\left(\nu_{g}+p\right) / 2\right]+2 \log \Gamma\left[\nu_{g} / 2\right]+p\left(\log \nu_{g}+\log \pi\right)-2 \log \pi_{g} \\
& +\left(\nu_{g}+p\right) \log \left[1+\frac{1}{\nu_{g}}\left(\left\|\boldsymbol{\mu}_{g}-P_{g}(\mathbf{x})\right\|_{\mathbf{A}_{g}}^{2}+\frac{1}{b_{g}}\left\|\mathbf{x}-P_{g}^{\prime}(\mathbf{x})\right\|^{2}\right)\right] \\
& +\sum_{j=1}^{d_{g}} \log a_{j g}+\left(p-d_{g}\right) \log b_{g} .
\end{aligned}
$$




\section{Bibliography}

Aitken, A. C. (1926). On Bernoulli's numerical solution of algebraic equations. Proceedings of the Royal Society of Edinburgh, 46, 289-305.

Andrews, J. L. and McNicholas, P. D. (2011a). Extending mixtures of multivariate t-factor analyzers. Statistics and Computing, 21(3), 361-373.

Andrews, J. L. and McNicholas, P. D. (2011b). Mixtures of modified t-factor analyzers for model-based clustering, classification, and discriminant analysis. Journal of Statistical Planning and Inference, 141(4), 1479-1486.

Andrews, J. L. and McNicholas, P. D. (2012). Model-based clustering, classification, and discriminant analysis via mixtures of multivariate t-distributions. Statistics and Computing, 22(5), 1021-1029.

Andrews, J. L. and McNicholas, P. D. (2014). teigen: Model-based clustering and classification with the multivariate t-distribution. $R$ package version, 1, 24-46.

Andrews, J. L., McNicholas, P. D., and Chalifour, M. (2015). mmtfa: Model-Based Clustering and Classification with Mixtures of Modified t Factor Analyzers. R package version 0.1 . 
Andrews, J. L., Wickins, J. R., Boers, N. M., and McNicholas, P. D. (2017). teigen: An $\mathrm{R}$ package for model-based clustering and classification via the multivariate $\mathrm{t}$ distribution. Journal of Statistical Software. To appear.

Baek, J. and McLachlan, G. J. (2011). Mixtures of common t-factor analyzers for clustering high-dimensional microarray data. Bioinformatics, 27, 1269-1276.

Banfield, J. D. and Raftery, A. E. (1993). Model-based Gaussian and non-Gaussian clustering. Biometrics, 49(3), 803-821.

Barndorff-Nielsen, O., Kent, J., and Sørensen, M. (1982). Normal variance-mean mixtures and z distributions. International Statistical Review / Revue Internationale de Statistique, 50(2), pp. 145-159.

Berge, L., Bouveyron, C., and Girard, S. (2012). HDclassif: An R package for modelbased clustering and discriminant analysis of high-dimensional data. Journal of Statistical Software, 46(6), 1-29.

Bibring, J.-P., Soufflot, A., Berthé, M., Langevin, Y., Gondet, B., Drossart, P., Bouyé, M., Combes, M., Puget, P., Semery, A., et al. (2004). Omega: Observatoire pour la minéralogie, l'eau, les glaces et l'activité. In Mars Express: The Scientific Payload, volume 1240, pages 37-49.

Biernacki, C., Celeux, G., and Govaert, G. (2000). Assessing a mixture model for clustering with the integrated completed likelihood. IEEE transactions on pattern analysis and machine intelligence, 22(7), 719-725. 
Bouveyron, C. and Brunet-Saumard, C. (2014). Model-based clustering of highdimensional data: A review. Computational Statistics and Data Analysis, 71, $52-78$.

Bouveyron, C., Girard, S., and Schmid, C. (2007). High-dimensional data clustering. Computational Statistics \& Data Analysis, 52(1), 502-519.

Browne, R. P. and McNicholas, P. D. (2015). A mixture of generalized hyperbolic distributions. Canadian Journal of Statistics, 43(2), 176-198.

Browne, R. P., ElSherbiny, A., and McNicholas, P. D. (2015). mixture: Mixture Models for Clustering and Classification. R package version 1.4.

Cattell, R. B. (1966). The scree test for the number of factors. Multivariate behavioral research, $\mathbf{1}(2), 245-276$.

Celeux, G. and Govaert, G. (1995). Gaussian parsimonious clustering models. Pattern recognition, 28(5), 781-793.

Dasgupta, A. and Raftery, A. E. (1998). Detecting features in spatial point processes with clutter via model-based clustering. Journal of the American Statistical Association, 93, 294-302.

Dempster, A. P., Laird, N. M., and Rubin, D. B. (1977). Maximum likelihood from incomplete data via the EM algorithm. Journal of the Royal Statistical Society: Series B, 39(1), 1-38.

Forina, M., Armanino, C., Castino, M., and Ubigli, M. (1986). Multivariate data analysis as a discriminating method of the origin of wines. Vitis, 25, 189-201. 
Fraley, C. and Raftery, A. E. (2002). Model-based clustering, discriminant analysis, and density estimation. Journal of the American statistical Association, 97(458), $611-631$.

Franczak, B. C., Browne, R. P., and McNicholas, P. D. (2014). Mixtures of shifted asymmetriclaplace distributions. Pattern Analysis and Machine Intelligence, IEEE Transactions on, 36(6), 1149-1157.

Ghahramani, Z. and Hinton, G. E. (1997). The EM algorithm for mixtures of factor analyzers. Technical report, Technical Report CRG-TR-96-1, University of Toronto.

Hubert, L. and Arabie, P. (1985). Comparing partitions. Journal of Classification, 2(1), 193-218.

Hurley, C. (2012). gclus: Clustering Graphics. R package version 1.3.1.

Kass, R. E. and Raftery, A. E. (1995). Bayes factors. Journal of the American Statistical Association, 90(430), 773-795.

Kass, R. E. and Wasserman, L. (1995). A reference bayesian test for nested hypotheses and its relationship to the schwarz criterion. Journal of the American Statistical Association, 90(431), 928-934.

Keribin, C. (2000). Consistent estimation of the order of mixture models. Sankhyā: The Indian Journal of Statistics, Series A, pages 49-66.

Kotz, S. and Nadarajah, S. (2004). Multivariate t-distributions and their applications. Cambridge University Press. 
Leroux, B. G. et al. (1992). Consistent estimation of a mixing distribution. The Annals of Statistics, 20(3), 1350-1360.

Lin, T.-I. (2010). Robust mixture modeling using multivariate skew t distributions. Statistics and Computing, 20(3), 343-356.

Lin, T.-I., McNicholas, P. D., and Hsiu, J. H. (2014). Capturing patterns via parsimonious t mixture models. Statistics and Probability Letters, 88, 80-87.

Lindsay, B. G. (1995). Mixture Models: Theory, Geometry, and Applications. Institute for Mathematical Statistics: Hayward, CA.

McLachlan, G. J. and Krishnan, T. (2008). The EM Algorithm and Extensions. Wiley, New York, 2nd edition.

McLachlan, G. J. and Peel, D. (2000). Finite Mixture Models. John Wiley and Sons, New York.

McLachlan, G. J., Bean, R., and Jones, L. B.-T. (2007). Extension of the mixture of factor analyzers model to incorporate the multivariate t-distribution. Computational Statistics 83 Data Analysis, 51(11), 5327-5338.

McNicholas, P. D. (2016a). Mixture Model-Based Classification. Chapman and Hall/CRC Press, Boca Raton.

McNicholas, P. D. (2016b). Model-based clustering. Journal of Classification, 33(3), $331-373$.

McNicholas, P. D. and Murphy, T. B. (2008). Parsimonious Gaussian mixture models. Statistics and Computing, 18(3), 285-296. 
McNicholas, P. D. and Murphy, T. B. (2010). Model-based clustering of microarray expression data via latent Gaussian mixture models. Bioinformatics, 26(21), 27052712 .

McNicholas, P. D., Murphy, T. B., McDaid, A. F., and Frost, D. (2010). Serial and parallel implementations of model-based clustering via parsimonious Gaussian mixture models. Computational Statistics and Data Analysis, 54(3), 711-723.

McNicholas, P. D., ElSherbiny, A., McDaid, A. F., and Murphy, T. B. (2015). pgmm: Parsimonious Gaussian Mixture Models. R package version 1.2.

McNicholas, S. M., McNicholas, P. D., and Browne, R. P. (2017). A mixture of variance-gamma factor analyzers. In S. E. Ahmed, editor, Big and Complex Data Analysis: Methodologies and Applications, pages 369-385. Springer International Publishing, Cham.

Meng, X.-L. and Rubin, D. B. (1993). Maximum likelihood estimation via the ECM algorithm: A general framework. Biometrika, 80(2), 267-278.

Murray, P. M., Browne, R. P., and McNicholas, P. D. (2014). Mixtures of skew-t factor analyzers. Computational Statistics and Data Analysis, 77(0), 326-335.

Murray, P. M., Browne, R. P., and McNicholas, P. D. (2017). A mixture of SDB skew-t factor analyzers. Econometrics and Statistics, 3, 160-168.

Peel, D. and McLachlan, G. J. (2000). Robust mixture modelling using the t distribution. Statistics and Computing, 10(4), 339-348.

Puts, M., Daas, P., and de Waal, T. (2015). Finding errors in big data. Significance, 12(3), 26-29. 
R Core Team (2016). R: A Language and Environment for Statistical Computing. R Foundation for Statistical Computing, Vienna, Austria.

Rand, W. M. (1971). Objective criteria for the evaluation of clustering methods. Journal of the American Statistical Association, 66, 846-850.

Schwarz, G. (1978). Estimating the dimension of a model. The Annals of Statistics, 6(2), 461-464.

Scott, D. W. and Thompson, J. R. (1983). Probability density estimation in higher dimensions. In Computer Science and Statistics: Proceedings of the fifteenth symposium on the interface, volume 528, pages 173-179. North-Holland, Amsterdam.

Steane, M. A., McNicholas, P. D., and Yada, R. (2012). Model-based classification via mixtures of multivariate t-factor analyzers. Communications in Statistics Simulation and Computation, 41(4), 510-523.

Vrbik, I. and Mcnicholas, P. D. (2014). Parsimonious skew mixture models for modelbased clustering and classification. Computational Statistics \&3 Data Analysis, 71, 196-210.

Wolfe, J. H. (1963). Object Cluster Analysis of Social Areas. Master's thesis, University of California, Berkeley. 